2000-2002), to model a hypothetical MVPA intervention scenario, simulating universal achievement of the government's MVPA target of 60 min (m) MVPA per day.

Methods 6344 children had MVPA (measured using accelerometers at 7 years[y]); of these, 4590 had data on outcome (CMHP), exposure (socio-economic circumstances) and potential confounders. CMHP at $11 \mathrm{y}$ were measured using parentreported Strengths and Difficulties Questionnaire (SDQ) total score, dichotomised using an established cut-off.

Predicted probabilities of CMHP were estimated in logistic marginal structural models, weighted for attrition, adjusted for MVPAand baseline and intermediate confounding (including externalising behaviours at $7[\mathrm{y}]$, to account for potential reverse causality between MVPA and some aspects of CMHP, such as hyperactivity).Inequalities were assessed using Risk ratios (RRs) and differences (RDs) [95\% CIs], according to household income quintiles. Intervention was simulated by reestimating predicted probabilities after modifying theMVPA variable.

Results 49\% of children achieved the $60 \mathrm{~m}$ MVPA target, with greater activity levels observed in lowest $(65 \mathrm{~m})$ compared to highest $(62 \mathrm{~m})$ income quintile. Greater MVPA was associated with increased risk of CMHP (RR 1.003 [0.9991.007]), and with externalising problems in particular (RR 1.009 [1.005-1.013]).

Overall prevalence of CMHP was $12.2 \%$, with relative and absolute inequalities between lowest and highest income quintiles (RR 2.6 [1.5-3.7]; RD 11.7 [6.0-17.4]).

Simulation of the intervention led to $96 \%$ achievement of the $60 \mathrm{~m} \mathrm{MVPA}$ target $(30 \mathrm{~m}$ average increase for all children, assuming $100 \%$ uptake). CMHP prevalence increased to 14.1\%. Relative inequality decreased slightly (RR 2.5 [1.5, 3.6]), and absolute inequality increased (RD 13.3\%; [6.6, 20.0]). In sensitivity analyses with internalising problems as the outcome, greater MVPA decreased absolute inequality in CMHP, but not relative risk or prevalence.

Conclusion Findings based on a UK-representative sample of children with objective MVPA data and a validated measure of CMHP, imply that universal achievement of the national MVPA target may not reduce prevalence in CMHP or absolute inequalities. However, these findings are likely subject to reverse causation (despite adjustment for earlier externalising behaviours). Further analyses will examine these relationships in detail, including differentiating aspects of CMHP (emotional, peer, conduct and hyperactivity subscales), using teacher-reported SDQ, and positive mental health outcomes.

\section{OP71 CAN YOU TURN ON A LIGHT SWITCH? EXPLORING WHETHER CHANGES TO DISABILITY-RELATED WELFARE PAYMENTS IN THE UK DISADVANTAGE CLAIMANTS WITH A MENTAL ILLNESS}

${ }^{1} \mathrm{KJ}$ Pybus ${ }^{*},{ }^{1} \mathrm{~K}$ Pickett, ${ }^{2} \mathrm{C}$ Lloyd, ${ }^{1} \mathrm{~S}$ Prady, ${ }^{1} \mathrm{R}$ Wilkinson. ${ }^{1}$ Department of Health Sciences, University of York, York, UK; ${ }^{2}$ Department of Social Policy and Social Work, University of York, York, UK

\subsection{6/jech-2018-SSMabstracts.70}

Background The experiences and outcomes of welfare claimants with a mental illness following recent welfare reforms in the United Kingdom (UK) have been explored as part of larger studies on the impact of such changes on people with disabilities but detailed evidence for this group of claimants is limited. This research explores the experiences and assessment outcomes of claimants with a mental illness undergoing changes to their disability-related welfare payments in the UK. Methods Experiences of welfare reform were first explored in interviews with claimants. Participants reported that they felt disadvantaged by having a mental illness when trying to obtain financial support compared to if they had a physical health condition (see results). This perception of differential outcomes was then explored through administrative data analysis.

Individuals with a mental illness $(n=18)$ living in Leeds, England were recruited via community support organisations and interviewed between January and April 2017. Data were analysed in Nvivo using a thematic analysis framework. The final sample consisted of individuals aged between 25-65, experiencing a range of mental health conditions including depression, anxiety, psychosis, bipolar affective disorder and borderline personality disorder.

Department of Work and Pensions administrative data for all claimants changing from Disability Living Allowance (DLA) to Personal Independence Payments (PIP) between 2013-2016 $(n=470,800)$ were then extracted to explore whether claimants with mental illness experienced differences in the level of financial support they received under the new system compared to claimants with non-psychiatric conditions. Odds ratios were calculated in Stata 15.1 to explore differences in assessment outcomes - whether existing financial support (DLA) increased, stayed the same or decreased under the new system (PIP) for claimants with psychiatric compared to nonpsychiatric conditions.

Results Interviewees reported that the updated eligibility criteria associated with changes to welfare payments did not appear to take account of the difficulties associated with mental health and described an assessment process focused primarily on physical capability. Further analysis using data on claimants transferring from DLA to PIP revealed that individuals with psychiatric conditions were 1.31 (95\% CI 1.29, 1.32) times more likely to have their financial support reduced or withdrawn entirely when transferring from DLA to PIP, compared to claimants with non-psychiatric conditions.

Conclusion These findings provide a starting point for exploring whether recent reforms to welfare payments in the UK have disadvantaged claimants with a mental illness and raise questions as to whether there is parity of esteem between mental and physical health in the welfare system. Given ongoing concerns regarding the marginalisation of people with mental illness, further research is needed to confirm these findings and to address any inequalities that may be present.

\section{OP72 BEING ALONE TOGETHER: A LONGITUDINAL DYADIC ANALYSIS ON THE IMPACT OF LONELINESS AND RELATIONSHIP QUALITY ON WELLBEING IN COUPLES COPING WITH DISABILITY} ${ }^{1,2} \mathrm{H}$ Tough*, ${ }^{1,2} \mathrm{MWG}$ Brinkhof, ${ }^{3} \mathrm{~J}$ Siegrist, ${ }^{1,2} \mathrm{C}$ Fekete. ${ }^{1}$ Social and Life Course
Epidemiology, Swiss Paraplegic Research, Nottwil, Switzerland; ${ }^{2}$ Health Sciences and Health
Policy, University of Luzern, Luzern, Switzerland; ${ }^{3}$ Faculty of Medicine, University of
Düsseldorf, Düsseldorf, Germany

\subsection{6/jech-2018-SSMabstracts.71}

Background Couples coping with disability are at an increased risk of experiencing poor wellbeing, this may be due to the potentially harmful effects of loneliness and poor relationship quality that this population are frequently exposed to. Both 
loneliness and poor relationship quality have profoundly detrimental effects on health and wellbeing. The experiences of loneliness and poor relationship quality are though to a elicit a stress response, which when experienced chronically, can result in behavioural biases and negative physical and mental health outcomes. In comparison, the experience of high quality relationships can buffer the negative effects of other external stressors. To date, little is known about how the perceptions of loneliness and relationship quality within couples coping with a disability affect wellbeing, or how the partners' perceptions of loneliness and relationship quality affect the individuals' wellbeing, furthermore the directionality of effect is not well understood.

Methods In this study, we investigate the actor and partner effects, and the reciprocal effects of loneliness and relationship quality on wellbeing. The analyses are based on longitudinal dyadic data from the pro-WELL study $(n=246)$, a Swiss community survey of persons with spinal cord injury (SCI) and their partners. Data was collected over a 12 month period using telephone interviews and questionnaires. We employed mixed effects modelling to explore actor and partner effects, and used cross-lagged path analysis to explore reciprocal effects. Both of these analyses were stratified to account for the potential differential effects in persons with SCI and their caregiving partners.

Results We found loneliness to be more prevalent in persons with SCI compared to their caregiving partners. Caregiving partners were found to be more sensitive to their own, and their partners' experiences of relationship quality, as we found significant actor effects $(\beta=0.15 \mathrm{CI}: 0.04,0.26)$, and significant partner effects of relationship quality on wellbeing $(\beta=0.21$ CI:0.10, 0.32). In both persons with SCI $(\beta=-0.30$ CI: $-0.41,-0.18)$ and their caregiving $(\beta=-0.20 \mathrm{CI}:-0.31,-$ $0.10)$, we found significant actor effects of loneliness on wellbeing. Over time, loneliness demonstrated reciprocal associations with wellbeing, as did relationship quality in caregiving partners, indicating a possible cycle of positive development between reducing loneliness, improving relationship quality and enhancing wellbeing.

Conclusion The findings of our study emphasise the high prevalence of loneliness in persons with SCI and the importance of reducing loneliness and strengthening relationship quality in order to improve wellbeing in couples coping with disability.

\section{OP73 THE IMPACT OF MAJOR MENTAL ILLNESS ON RISK OF STROKE AND MYOCARDIAL INFARCTION IN PEOPLE WITH TYPE 2 DIABETES IN SCOTLAND: AN ANALYSIS OF ROUTINELY COLLECTED HEALTH DATA}

\begin{abstract}
${ }^{1,2} \mathrm{~K}$ Fleetwood*, ${ }^{1,2} \mathrm{~S}$ Wild, ${ }^{3} \mathrm{D}$ Smith, ${ }^{4} \mathrm{~K}$ Licence, ${ }^{3} \mathrm{~S}$ Mercer, ${ }^{1} \mathrm{C}$ Sudlow, ${ }^{1,2} \mathrm{C}$ Jackson. ${ }^{1}$ Usher Institute of Population Health Sciences and Informatics, University of Edinburgh, Edinburgh, UK; ${ }^{2}$ On behalf of the Scottish Diabetes Research Network Epidemiology Group; ${ }^{3}$ Institute of Health and Wellbeing, University of Glasgow, Glasgow, UK; ${ }^{4}$ Public Health and Intelligence, NHS National Services Scotland, Edinburgh, UK
\end{abstract}

10.1136/jech-2018-SSMabstracts.72

Background In Scotland, major mental illness reduces life expectancy by approximately 17 years, with cardiovascular disease (CVD) the leading cause of death. We aimed to investigate whether history of major mental illness affects CVD risk in people with type 2 diabetes mellitus (T2DM) in Scotland.
Methods We identified adults diagnosed with T2DM between 2004 and 2016 from a national diabetes register (the Scottish Care Information - Diabetes database). We ascertained history of mental illness from psychiatric and acute hospital admission records and incident/recurrent stroke or myocardial infarction (abbreviated to CVD) from acute hospital admission and mortality records. Using Cox regression analysis, we obtained hazard ratios (HRs) for CVD risk among people with a history of schizophrenia, bipolar disorder or depression, compared to those with T2DM but without a history of mental illness.

Results We included 212011 people with T2DM. Of these, 2107 (1.0\%) had schizophrenia, 1521 (0.7\%) had bipolar disorder and $5288(2.5 \%)$ had depression. People with these major mental disorders were younger at diagnosis of T2DM and had higher prevalence of smoking and history of alcohol use disorders than the comparison group. After adjusting for age, sex, area-based deprivation, hypertension and previous history of CVD, HRs (95\% confidence interval) were 1.27 $(1.05,1.53)$ for schizophrenia, $1.45(1.23,1.70)$ for bipolar disorder and $1.50(1.37,1.65)$ for depression compared to those without a history of mental illness. Additional adjustment for smoking and alcohol use disorder attenuated effect estimates to $1.12(0.93,1.36)$ for schizophrenia, 1.35 (1.15, 1.59) for bipolar disorder and $1.37(1.25,1.51)$ for depression.

Conclusion Schizophrenia, bipolar disorder and depression are associated with increased CVD risk among people with T2DM and this is partially explained by higher prevalence of smoking and alcohol use disorders in people with these conditions. This highlights the need for better CVD prevention in people with T2DM and major mental illness especially with respect to modifiable risk factors. Major strengths are that the diabetes register includes $99 \%$ of all people with diabetes in Scotland and the follow-up period is relatively long. However, our definition of mental illness was limited to hospital admission data only and so findings may not apply to people with mental illness not admitted to hospital. Future analyses will also include adjustment for psychotropic medication use.

\section{Health behaviours in young people}

\section{OP74 EXPLORING STUDENTS' PERCEPTIONS OF AND EXPERIENCES WITH ALCOHOL DURING THE TRANSITION TO UNIVERSITY AND ACROSS THE FIRST YEAR}

${ }^{1} \mathrm{AC}$ Fuller*, ${ }^{1} \mathrm{R}$ Murray, ${ }^{2} \mathrm{KM}$ Fleming, ${ }^{1} \mathrm{~L}$ Szatkowski, ${ }^{1} \mathrm{M}$ Bains. ${ }^{1}$ Epidemiology and Public Health, University of Nottingham, Nottingham, UK; ${ }^{2}$ Public Health and Policy, University of Liverpool, Liverpool, UK

\subsection{6/jech-2018-SSMabstracts.73}

Background Recent studies suggest binge drinking rates are high among university students in the UK. It has been suggested that many students arrive at university with established drinking patterns, but that freshers' week (FW) also serves as an introduction to the university drinking culture, and there are many non-drinkers who may feel excluded from this. The aim of this study was to explore the extent to which students' views of and experiences with alcohol change as they transition to university and across their first academic year.

Methods First year undergraduates entering a large UK university in September 2016 who completed an online Students' Union welfare survey in August 2016 were invited to 